\title{
Alterations of Brain Energy Metabolism in Type 2 Diabetic Goto-Kakizaki Rats Measured In Vivo by ${ }^{13} \mathrm{C}$ Magnetic Resonance Spectroscopy
}

\author{
Freya-Merret Girault $^{1} \cdot$ Sarah Sonnay $^{1} \cdot$ Rolf Gruetter $^{1,2,3}$ • João M. N. Duarte ${ }^{1}$
}

Received: 13 June 2017 /Revised: 21 August 2017 / Accepted: 20 September 2017 /Published online: 2 October 2017

(C) Springer Science+Business Media, LLC 2017

\begin{abstract}
Type 2 diabetes (T2D) is associated with deterioration of brain structure and function. Here, we tested the hypothesis that $\mathrm{T} 2 \mathrm{D}$ induces a reorganization of the brain metabolic networks that support brain function. For that, alterations of neuronal and glial energy metabolism were investigated in a T2D model, the Goto-Kakizaki (GK) rat. ${ }^{13} \mathrm{C}$ magnetic resonance spectroscopy in vivo at $14.1 \mathrm{~T}$ was used to detect ${ }^{13} \mathrm{C}$ labeling incorporation into carbons of glutamate, glutamine, and aspartate in the brain of GK $(n=7)$ and Wistar $(n=13)$ rats during intravenous $\left[1,6-{ }^{13} \mathrm{C}\right]$ glucose administration. Labeling of brain glucose and amino acids over time was analyzed with a twocompartment mathematical model of brain energy metabolism to determine the rates of metabolic pathways in neurons and glia. Compared to controls, GK rats displayed lower rates of brain glutamine synthesis $(-32 \%$, $P<0.001)$ and glutamate-glutamine cycle $(-40 \%$, $\mathrm{P}<0.001$ ), and mitochondrial tricarboxylic acid (TCA) cycle rate in neurons $(-7 \%, P=0.036)$. In contrast, the TCA cycle rate of astrocytes was larger in GK rats than controls $(+21 \%, P=0.042)$. We conclude that T2D alters brain energy metabolism and impairs the glutamateglutamine cycle between neurons and astrocytes, in line
\end{abstract}

João M. N. Duarte

joao.duarte@epfl.ch

1 Laboratory for Functional and Metabolic Imaging (LIFMET), École Polytechnique Fédérale de Lausanne (EPFL), Station 6, 1015 Lausanne, CH, Switzerland

2 Department of Radiology, University of Lausanne, Lausanne, Switzerland

3 Department of Radiology, University of Geneva, Geneva, Switzerland with diabetes-induced neurodegeneration and astrogliosis underlying brain dysfunction.

Keywords Insulin resistance $\cdot$ Glucose $\cdot$ Brain energy metabolism $\cdot$ Magnetic resonance spectroscopy $\cdot$ Neuron-glia interactions

\begin{tabular}{|c|c|}
\hline \multicolumn{2}{|c|}{ Abbreviations } \\
\hline $\mathrm{CMR}_{\mathrm{glc}(\mathrm{ox})}$ & Oxidative fraction of $\mathrm{CMR}_{\mathrm{glc}}$ \\
\hline $\mathrm{CMR}_{\mathrm{glc}}$ & Cerebral metabolic rate of glucose \\
\hline FE & Fractional enrichment \\
\hline GTT & Glucose tolerance test \\
\hline$K_{\mathrm{t}}$ & $\begin{array}{l}\text { Apparent Michaelis constant of glucose } \\
\text { transport }\end{array}$ \\
\hline MRS & Magnetic resonance spectroscopy \\
\hline $\mathrm{T} 2 \mathrm{D}$ & Type 2 diabetes \\
\hline TCA cycle & Tricarboxylic acid cycle \\
\hline$V_{\text {dil }}$ & Dilution of glial acetyl-CoA \\
\hline$V_{\mathrm{g}}$ & $\begin{array}{l}\text { Rate of glial TCA cycle resulting in full } \\
\text { pyruvate oxidation }\end{array}$ \\
\hline$V_{\mathrm{GS}}$ & Rate of glutamine synthetase \\
\hline$V_{\text {in }}$ & Dilution of pyruvate \\
\hline$V_{\mathrm{NT}}$ & $\begin{array}{l}\text { Rate of apparent glutamatergic } \\
\text { neurotransmission }\end{array}$ \\
\hline VOI & Volume of interest \\
\hline$V_{\text {out }}$ & Pyruvate efflux \\
\hline$V_{\mathrm{PC}}$ & Rate of pyruvate carboxylase \\
\hline$V_{\mathrm{TCA}}^{\mathrm{g}}$ & Glial TCA cycle \\
\hline$V_{\mathrm{TCA}}{ }^{\mathrm{n}}$ & Neuronal TCA cycle \\
\hline$V_{\mathrm{X}}^{\mathrm{g}}$ & Rate of oxoacid-amino acid exchange in glia \\
\hline$V_{\mathrm{X}}^{\mathrm{n}}$ & $\begin{array}{l}\text { Rate of oxoacid-amino acid exchange } \\
\text { in neurons }\end{array}$ \\
\hline ZDF & Zucker diabetic fatty \\
\hline
\end{tabular}




\section{Introduction}

Type 2 diabetes (T2D) is associated with cognitive decrements and increased risk for dementia (Biessels et al. 2006; Spauwen et al. 2013; Baumgart et al. 2015; Geijselaers et al. 2015). Importantly, it has been established that a broad range of cognitive domains are affected by T2D (Messier 2005; van den Berg et al. 2008; Palta et al. 2014), which suggests a rather widespread impact on the central nervous system, called diabetic encephalopathy.

Metabolic syndrome patients without clearly installed T2D may also display cognitive decrements (van den Berg et al. 2008). Indeed, chronic hyperglycemia, microvascular complications, insulin resistance, dyslipidemia, and hypertension occur in diabetes, and are all important risk factors for cognitive dysfunction (Duarte 2015; Moheet et al. 2015). This multiplicity of factors with potential impact on the central nervous system makes the mechanisms of diabetes-induced brain dysfunction difficult to study. Nevertheless, it is well established that diabetic encephalopathy derives from cellular damage caused by both glucose neurotoxicity upon uncontrolled hyperglycemia and from defective brain insulin signaling by either insulin deficiency or receptor desensitization (Tomlinson and Gardiner 2008; Rönnemaa et al. 2008). Studies in a plethora of rodent models of diabetes have contributed to elucidate cellular derangements in the diabetic brain (Duarte 2015). In particular, diabetic conditions lead to synaptic deterioration that results in defective neurotransmission and synaptic plasticity, which directly have functional consequences (Nitta et al. 2002; Duarte et al. 2009, 2012; Calvo-Ochoa et al. 2014). Furthermore, this neurodegenerative process is accompanied by neuroinflammation and astrogliosis (Saravia et al. 2002; Baydas et al. 2003; Duarte et al. 2009, 2012; Calvo-Ochoa et al. 2014). Interestingly, intranasal insulin treatment in insulin-deficient mice was shown to ameliorate synaptic degeneration and deficits in learning and memory, without preventing hyperglycemia (Francis et al. 2008). This indicates that impairment of central insulin signaling is indeed an important factor for diabetesinduced brain injury.

Progress in magnetic resonance imaging (MRI) techniques has led to a growing number of human studies investigating functional and structural cerebral changes in diabetes (Moheet et al. 2009). In parallel, studies with magnetic resonance spectroscopy (MRS), which is a non-invasive technique to quantify metabolite profiles, have generally identified reduced levels of the putative neuronal marker $N$-acetylaspartate, as well as increased in myo-inositol content that has been proposed to be a marker of astrocyte density (Duarte 2015). Accordingly, altered metabolite profiles have been also reported in the brain of diabetes models (van der Graaf et al. 2004; Duarte et al. 2009; Wang et al. 2012). However, such measurements cannot be used to infer on alterations of brain energy metabolism pathways, which are likely to occur before irreversible tissue damage occurs, namely tissue atrophy.

The adult brain requires a steady supply of oxygen and glucose, and disturbance of their metabolism was suggested to be notably associated with accelerated cognitive impairment (Kapogiannis and Mattson 2011). Accordingly, insulin resistance has been proposed to be directly associated with reduced cortical glucose consumption and cognitive impairment (Baker et al. 2011). On the other hand, impaired mitochondrial metabolism that results in an energetic deficit and in increased oxidative stress has been proposed as a shared mechanistic link between Alzheimer's disease and diabetes (Moreira et al. 2007). In this study, we tested the hypothesis that insulin-resistant diabetes involves a rearrangement of the metabolic pathways that fuel brain function.

Pathways of energy metabolism can be studied in the living brain by ${ }^{13} \mathrm{C}$ MRS during administration of ${ }^{13} \mathrm{C}$-enriched substrates (Lanz et al. 2013). By measuring ${ }^{13} \mathrm{C}$ isotope incorporation over time into specific carbon positions of different molecules, and by analyzing these ${ }^{13} \mathrm{C}$ enrichments over time with appropriate mathematical models, one can determine the rates of oxidative metabolism in neurons and astrocytes, as well as their metabolic interactions, namely the glutamateglutamine cycle rate (Duarte et al. 2011; Sonnay et al. 2016). Using state-of-the-art ${ }^{13} \mathrm{C}$ MRS at high magnetic field, we measured ${ }^{13} \mathrm{C}$ incorporation from glucose into brain amino acids in order to determine fluxes through pathways of energy metabolism, and the glutamate-glutamine cycle in a diabetes model, the Goto-Kakizaki (GK) rat. The GK rat is a wellcharacterized nonobese, non-ketotic T2D model derived from outbred, glucose-intolerant Wistar rats (Portha 2005), which were used as controls.

\section{Materials and Methods}

\section{Chemicals}

Isoflurane and $\alpha$-chloralose were purchased from Animalcare (York, UK) and Acros Organics (Geel, Belgium), respectively. ${ }^{2} \mathrm{H}_{2} \mathrm{O}\left(99.9 \%{ }^{2} \mathrm{H}\right),\left[1,6-{ }^{13} \mathrm{C}\right]$ glucose $\left(99.9 \%{ }^{13} \mathrm{C}\right.$ in both carbons) and other chemicals were obtained from Sigma-Aldrich (Buchs, Switzerland).

\section{Animals}

All experiments were performed in accordance with the Swiss federal law on animal experimentation and approved by the local authority (EXPANIM-SCAV), and are reported according to the ARRIVE guidelines. Sample size estimation was based on previous work (Duarte et al. 2011; Sonnay et al. 2016). Male GK $(n=7)$ and Wistar rats $(n=13)$ were purchased from Charles River Laboratoires (L'Arbresle, France) 
and housed in pairs in a room at $22{ }^{\circ} \mathrm{C}, 50-60 \%$ humidity, and a 12-h light-dark cycle. Food (Kliba Nafag 3800, Provimi Kliba, Kaiseraugst, Switzerland) and water were provided ad libitum. The GK rat model of T2D was chosen because it shows mild hyperglycemia and is devoid of obesity. The diabetic phenotype is installed at 2-3 months of age (Portha 2005). This study focused on male rats since the differential steroid hormone profiles of male and female GK rats impacts the brain over diabetes progression (Candeias et al. 2016). MRS experiments were performed between 6 and 7 months of age. One week prior MRS, a glucose tolerance test (GTT) was performed after $16 \mathrm{~h}$ fasting. Briefly, a $30-\mu \mathrm{L}$ blood sample was collected from the tail tip, and serum was stored to measure glucose, insulin, and corticosterone. Then, animals were weighted and injected i.p with $2 \mathrm{~g} / \mathrm{kg}$ of glucose prepared as $30 \%(w / v)$ in saline. Glycemia was measured from tail blood immediately before and up to $4 \mathrm{~h}$ after glucose administration using the Ascencia Contour glucometer (Bayer, Zürich, Switzerland).

\section{MRS}

Animal preparation was detailed previously (Duarte et al. 2009). Briefly, after overnight fasting ( $8-10 \mathrm{~h}$ ), rats were anesthetized with $2 \%$ isoflurane vaporized in $30 \% \mathrm{O}_{2}$ in air, and were intubated and mechanically ventilated. Catheters were inserted into a femoral vein for infusion of saline solutions containing $\left[1,6-{ }^{13} \mathrm{C}\right]$ glucose or $\alpha$-chloralose, and into a femoral artery for blood sampling and physiology monitoring. Heart rate, arterial blood pressure, body temperature, and breathing rate were recorded with the SA Instruments monitoring system (Stony Brook, NY, USA). Arterial $\mathrm{pH}$ and pressures of $\mathrm{CO}_{2}\left(\mathrm{P}_{\mathrm{a}} \mathrm{CO}_{2}\right)$ and $\mathrm{O}_{2}\left(\mathrm{P}_{\mathrm{a}} \mathrm{O}_{2}\right)$ were measured using the AVL Compact 3 blood analyzer (Roche Diagnostics, Rotkreuz, Switzerland), and were maintained at physiological levels by adjusting respiratory rate and volume. Body temperature was maintained at $37{ }^{\circ} \mathrm{C}$ by warm water circulation. Plasma glucose and lactate were determined with a Reflotron Plus system (Roche) and a GM7 Micro-Stat analyzer (Analox Instruments, London, UK), respectively. After positioning the animals in a homebuilt holder with ear and mouth inserts for head fixation, anesthesia was switched to $\alpha$ chloralose $(80 \mathrm{mg} / \mathrm{kg}$ bolus i.v. followed by continuous infusion at $27 \mathrm{mg} / \mathrm{kg} / \mathrm{h}$ ). For ${ }^{13} \mathrm{C}$ MRS, $\left[1,6-{ }^{13} \mathrm{C}\right]$ glucose was administered i.v. to reach $70 \%$ of fractional enrichment (FE) in plasma within $5 \mathrm{~min}$ and throughout the whole experiment (Duarte et al. 2011).

All experiments were performed on a 14.1-T/26-cm horizontal bore magnet (Magnex Scientific, Abingdon, UK) with 12 -cm gradients $(400 \mathrm{mT} / \mathrm{m}$ in $120 \mu \mathrm{s})$, interfaced to a Direct Drive console (Agilent Technologies, Palo Alto, CA, USA). A homebuilt surface coil consisting of a ${ }^{1} \mathrm{H}$ quadrature coil and a ${ }^{13} \mathrm{C}$ linearly polarized coil was used for transmission and signal reception (Duarte et al. 2011). The rat brain was placed at the isocenter of the magnet and $\mathrm{T}_{2}$-weighted images were acquired for anatomical reference. A volume of interest (VOI) of $\sim 400 \mu \mathrm{L}$ was placed in the brain, encompassing the cortex, hippocampus, thalamus, and striatum. After FAST(EST)MAP shimming, localized ${ }^{1} \mathrm{H}$ spectra were acquired using STEAM with echo time of $2.8 \mathrm{~ms}$, mixing time of $20 \mathrm{~ms}$, and repetition time of $4 \mathrm{~s}$ and ${ }^{13} \mathrm{C}$ spectra were acquired using the semiadiabatic DEPT combined with 3D-ISIS ${ }^{1} \mathrm{H}$ localization and outer-volume suppression as detailed elsewhere (Henry et al. 2003). In particular, the last ${ }^{1} \mathrm{H}$ of DEPT was set to $45^{\circ}$ to allow simultaneous detection of $\mathrm{CH}_{3}, \mathrm{CH}_{2}$, and $\mathrm{CH}$; the inter-pulse delay was optimized for $J_{\mathrm{CH}}=130 \mathrm{~Hz}$; the three ${ }^{13} \mathrm{C}$ pulses were adiabatic, consisting of a 2 -ms segmented $0^{\circ}$ BIR-4 pulse (for details, see Henry et al. 2003). Spectra were analyzed using LCModel (Stephen Provencher Inc., Oakville, $\mathrm{ON}$, Canada), and ${ }^{13} \mathrm{C}$ labeling was converted to concentration as detailed previously (Duarte and Gruetter 2013).

\section{Serum and Plasma Analyses}

Samples were stored at $-80^{\circ} \mathrm{C}$, and then analyzed simultaneously. Insulin concentration was determined using the Rat Insulin ELISA kit from Mercodia (Uppsala, Sweden). Serum corticosterone and plasma leptin were assayed with ELISA kits from Abcam (Cambridge, UK). For determination of metabolite concentrations and $\mathrm{FE},{ }^{1} \mathrm{H}$ spectra were acquired from plasma samples diluted 1:10 in ${ }^{2} \mathrm{H}_{2} \mathrm{O}$ using a DRX-600 spectrometer with a 5-mm cryoprobe (Bruker BioSpin, Falländen, Switzerland) and quantified as previously (Duarte et al. 2007).

\section{Metabolic Modeling and Statistical Analyses}

Fluxes of brain energy metabolism were estimated with the two-compartment model described previously (Duarte et al. 2011). Glucose transport and consumption were analyzed by fitting both dynamic and steady-state MRS data with a reversible Michaelis-Menten model (Duarte and Gruetter 2012). The model was firstly fitted to glucose concentrations measured at steady-state in the brain and plasma. The resulting apparent Michaelis constant of glucose transport $K_{\mathrm{t}}$ was then used in the analysis of the average curves of glucose C6 labeling (Sonnay et al. 2016), which allowed to determine the apparent maximum transport rate $\left(T_{\max }\right)$ and the cerebral metabolic rate of glucose $\left(\mathrm{CMR}_{\mathrm{glc}}\right)$. The mathematical model (Duarte et al. 2011) was then adjusted to the average ${ }^{13} \mathrm{C}$ enrichment curves of glutamate, glutamine, and aspartate to estimate fluxes through the apparent glutamatergic neurotransmission (i.e., glutamate-glutamine cycle, $V_{\mathrm{NT}}$ ), neuronal tricarboxylic acid (TCA) cycle $\left(V_{\mathrm{TCA}}{ }^{\mathrm{n}}\right)$, the fraction of the glial TCA cycle that results in full oxidation of pyruvate $\left(V_{\mathrm{g}}\right)$, pyruvate carboxylase $\left(V_{\mathrm{PC}}\right)$, labeling exchange between oxaloacetate and aspartate and between 2-oxoglutarate and 
glutamate $\left(V_{\mathrm{X}}^{\mathrm{n}}\right.$ in neurons and $V_{\mathrm{X}}^{\mathrm{g}}$ in glia), pyruvate efflux $\left(V_{\text {out }}\right)$, and dilution of glial acetyl-CoA $\left(V_{\text {dil }}\right)$. The model includes the following calculated fluxes: glutamine synthetase $\left(V_{\mathrm{GS}}=V_{\mathrm{NT}}+V_{\mathrm{PC}}\right)$, glial TCA cycle $\left(V_{\mathrm{TCA}}^{\mathrm{g}}=V_{\mathrm{g}}+V_{\mathrm{PC}}\right)$, $\mathrm{CMR}_{\text {glc (ox })}=\left(V_{\mathrm{TCA}}{ }^{\mathrm{n}}+V_{\mathrm{TCA}}{ }^{\mathrm{g}}+V_{\mathrm{PC}}\right) / 2$ that is the oxidative fraction of glucose metabolism, and dilution of pyruvate through $V_{\text {in }}=V_{\text {out }}+2 \mathrm{CMR}_{\text {glc(ox })}-2 \mathrm{CMR}_{\text {glc }}$. For the modeling, concentrations of glutamate, glutamine, and aspartate were determined in vivo, and concentrations of TCA cycle intermediates were assumed to be $0.1 \mu \mathrm{mol} / \mathrm{g}$ in any compartment (Duarte et al. 2011). Neurons were assumed to retain $90 \%$ of total glutamate and aspartate pools, while glial cells contain $90 \%$ of the total glutamine concentration. With the exception of glucose, metabolic but not isotopic steady-state was assumed over the time course of the experiment. Reliability of the model was evaluated by Monte-Carlo analysis, and the variance of each parameter was estimated from the respective probability distribution of 1000 simulations (Duarte et al. 2011). Error propagation was taken into account in all calculations. Fluxes are shown with associated SD. Flux comparison between experimental groups was performed by permutation analysis followed by individual two-tailed Student $t$ tests (Sonnay et al. 2016). Reported $P$ values were corrected for multiple comparisons with the Bonferroni method. Remaining results are shown as mean \pm SEM, unless otherwise stated, and were also analyzed with two-tailed Student $t$ tests. Significance threshold was $P<0.05$.

\section{Results}

\section{Diabetic Phenotype of GK Rats}

Since multiple GK rat colonies are available (Portha 2005), we characterized the phenotype of the animals in this study. Fed glycemia was $5.1 \pm 0.2 \mathrm{mmol} / \mathrm{L}$ in controls and $8.4 \pm 0.5 \mathrm{mmol} /$ $\mathrm{L}$ in GK rats $(P<0.001)$. Rats were then fasted for $16 \mathrm{~h}$ for a glucose tolerance test. After fasting, body weight was $463 \pm 27$ and $314 \pm 8 \mathrm{~g}(P<0.001)$, glycemia was $3.4 \pm 0.1$ and $4.7 \pm 0.2 \mathrm{mmol} / \mathrm{L}(\mathrm{P}<0.001$, Fig. 1a), serum insulin was $0.20 \pm 0.05$ and $0.42 \pm 0.14 \mu \mathrm{g} / \mathrm{L}(P=0.130$, Fig. $1 b)$, and serum corticosterone was $37 \pm 6$ and $89 \pm 20 \mu \mathrm{g} / \mathrm{L}(P=0.042$, Fig. 1c) in Wistar and GK rats, respectively. Glycemia at the moment of glucose administration was taken as baseline for the GTT (Fig. 1d). Upon glucose administration, Wistar rats readily cleared the glucose from circulation, while GK rats took $\sim 4 \mathrm{~h}$ to return to baseline glycemia (Fig. 1d). Indeed, the area under the curve representing the glycemia rise above baseline was $46.2 \pm 4.1 \mathrm{mmol} . \mathrm{h} / \mathrm{L}$ for $\mathrm{GK}$ rats and only $13.4 \pm 2.0 \mathrm{mmol} . \mathrm{h} / \mathrm{L}$ for the controls $(P<0.001$, Fig. 1e).

After overnight fasting, ${ }^{13} \mathrm{C}$ MRS experiments were performed with $\left[1,6-{ }^{13} \mathrm{C}\right]$ glucose infusion to raise the plasma ${ }^{13} \mathrm{C}$ enrichment with a hyperglycemic clamp (Fig. 1F). The rate of glucose infusion at the end of the experiment, which can be taken as indicative of the steady-state whole body glucose disposal rate, was $10.4 \pm 0.6$ and $8.8 \pm 0.5 \mathrm{mmol} / \mathrm{kg} / \mathrm{h}$ in Wistar and GK rats, respectively $(P=0.036$, Fig. $1 \mathrm{~g})$. Plasma lactate concentration was larger in GK than Wistar rats at the end of the experiment $(4.3 \pm 0.2$ versus $3.0 \pm 0.3 \mathrm{mmol} / \mathrm{L}, P=0.003$, Fig. 1h). Before infusion, fasting plasma insulin was lower in Wistar than GK rats $(0.78 \pm 0.06$ versus $1.57 \pm 0.42 \mu \mathrm{g} / \mathrm{L}$, $P=0.013)$. Upon glucose administration, GK rats appeared to have a slower release of insulin into the circulation but, at steady-state, the glucose-stimulated rise of plasma insulin was larger in diabetic rats (Fig. 1i), namely reaching $26.3 \pm 4.5 \mu \mathrm{g} / \mathrm{L}$ in GK rats and $14.2 \pm 2.4 \mu \mathrm{g} / \mathrm{L}$ in controls after $4 \mathrm{~h}$ of glucose infusion $(P=0.023)$. A plasma sample prior to $\left[1,6-{ }^{13} \mathrm{C}\right]$ glucose infusion was further used to determine leptin concentration, which was similar in both groups $(1.3 \pm 0.4 \mu \mathrm{g} / \mathrm{L}$ in controls, $0.7 \pm 0.2 \mu \mathrm{g} / \mathrm{L}$ in GK rats, $P=0.226$ ).

\section{Labeling of Plasma Metabolites upon $\left[1,6{ }^{13} \mathrm{C}\right]$ Glucose Infusion}

Physiology parameters were within their normal range during the experiment (Table 1). Infusion of $\left[1,6-{ }^{13} \mathrm{C}\right]$ glucose resulted in labeling of glucose, lactate, alanine, and acetate. At the end of the ${ }^{13} \mathrm{C}$ MRS experiment, $\mathrm{FE}$ of glucose $\mathrm{C} 1$ was $0.671 \pm 0.002$ and $0.681 \pm 0.005$ in Wistar and GK rats, respectively $(P=0.080)$. Lactate $\mathrm{C} 3 \mathrm{FE}$ reached $0.426 \pm 0.004$ in Wistar and $0.389 \pm 0.008$ in GK rats $(P=0.003)$. In Wistar and GK rats, respectively, FE of alanine $\mathrm{C} 3$ was $0.482 \pm 0.007$ and $0.384 \pm 0.010(P<0.001)$, and $\mathrm{FE}$ of acetate $\mathrm{C} 2$ was $0.125 \pm 0.006$ and $0.122 \pm 0.008$ $(P=0.768)$. While glucose and lactate were abundant in plasma, alanine was only $0.18 \pm 0.01 \mathrm{mmol} / \mathrm{L}$ in Wistar and $0.33 \pm 0.01 \mathrm{mmol} / \mathrm{L}$ in $\mathrm{GK}$ rats $(P<0.001)$. Acetate was $1.08 \pm 0.07 \mathrm{mmol} / \mathrm{L}$ in Wistar and $0.86 \pm 0.06 \mathrm{mmol} / \mathrm{L}$ in GK rats $(P=0.028)$. Although $\beta$-hydroxybutyrate was quantifiable in ${ }^{1} \mathrm{H}$ spectra from the plasma of GK $(31 \pm 3 \mu \mathrm{mol} / \mathrm{L})$ and Wistar $(28 \pm 3 \mu \mathrm{mol} / \mathrm{L})$ rats $(P=0.489)$, its ${ }^{13} \mathrm{C}$ enrichment was undetectable. Noteworthy, these alterations of ${ }^{13} \mathrm{C}$ labeling in blood metabolites due to different peripheral metabolism in GK and Wistar rats are accounted for in the model of brain energy metabolism (Duarte et al. 2011).

\section{Brain Energy Metabolism by ${ }^{13} \mathrm{C}$ MRS}

Labeling of glucose, glutamate, glutamine, and aspartate in the brain was measured with a temporal resolution of about 5 min (Fig. 2a). Spectral differences include, e.g., smaller glutamine signals in GK than Wistar rats (Fig. 2b).

A stationary analysis of brain and plasma glucose concentrations was employed to determine the ratio of $T_{\max }$ to $\mathrm{CMR}_{\text {glc }}$ and $K_{\mathrm{t}}$ for glucose uptake into the brain (Fig. 3a). For Wistar rats, $T_{\max } / \mathrm{CMR}_{\mathrm{glc}}$ was $2.5 \pm 0.2$ and $K_{\mathrm{t}}$ was 
a

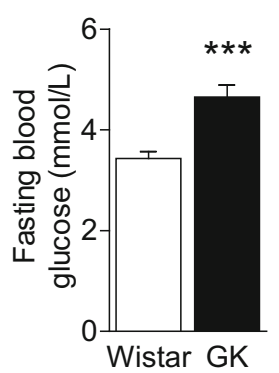

b

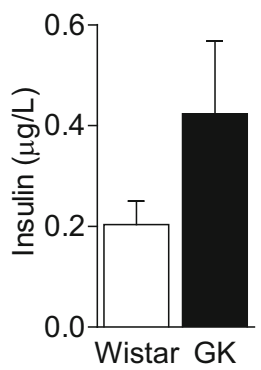

C

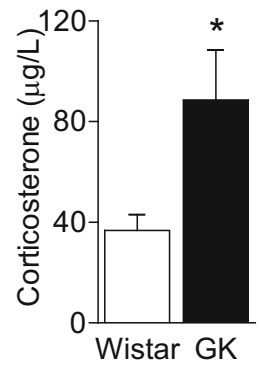

d

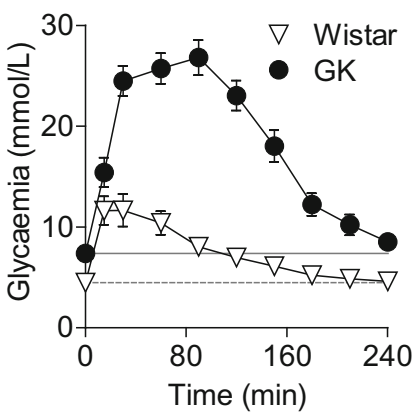

e

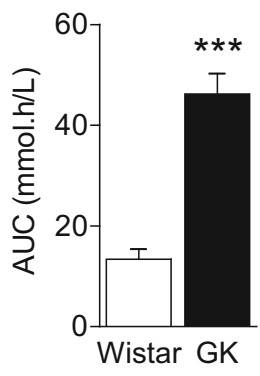

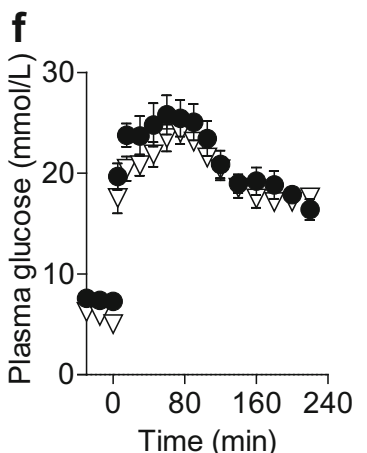

g

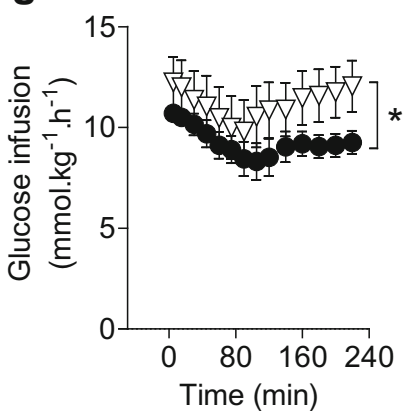

Fig. 1 Diabetic phenotype of GK rats. Blood glucose (a), serum insulin (b), and corticosterone (c) in GK and Wistar rats after 16-h fasting. A GTT shows impaired glucose clearance from circulation in GK rats (d), with an area under the curve (AUC) that was over three times larger than in Wistar rats (e). In the ${ }^{13} \mathrm{C}$ MRS experiment, plasma glucose was clamped at similar levels in GK and Wistar rats (f). In the steady-state

$4.3 \pm 2.1 \mathrm{mmol} / \mathrm{L}$. Similar values were estimated for the brain of GK rats: $T_{\max } / \mathrm{CMR}_{\mathrm{glc}}=2.5 \pm 0.3$ and $K_{\mathrm{t}}=5.0 \pm 2.9 \mathrm{mmol} /$ L. Then, the value of $K_{\mathrm{t}}$ for each experimental group was used to constrain the dynamic fit of brain $\left[{ }^{13} \mathrm{C}\right]$ glucose measured in vivo, thus allowing the determination of $T_{\max }$ and $\mathrm{CMR}_{\mathrm{glc}}$ (Fig. 3b). $T_{\max }$ was $1.06 \pm 0.04$ and $0.91 \pm 0.03 \mu$ mol.g. min $^{-1}$ $(P<0.001)$, and $\mathrm{CMR}_{\mathrm{glc}}$ was $0.40 \pm 0.02$ and

Table 1 Mean physiology parameters across the ${ }^{13} \mathrm{C}$ MRS experiment

\begin{tabular}{lcc}
\hline & Wistar & GK \\
\hline Temperature $\left({ }^{\circ} \mathrm{C}\right)$ & $37.3 \pm 0.1$ & $37.3 \pm 0.1$ \\
Heart rate $(\mathrm{bpm})$ & $348 \pm 14$ & $313 \pm 8$ \\
Blood pressure $(\mathrm{mm} \mathrm{Hg})$ & $152 \pm 12$ & $154 \pm 18$ \\
$\quad$ Systole & $96 \pm 8$ & $104 \pm 13$ \\
$\quad$ Dyastole & $99.2 \pm 0.2$ & $99.4 \pm 0.1$ \\
$\mathrm{O}_{2}$ saturation $(\%)$ & $7.33 \pm 0.01$ & $7.35 \pm 0.01$ \\
Arterial pH & $38 \pm 1$ & $35 \pm 1 *$ \\
$\mathrm{P}_{\mathrm{a}} \mathrm{CO}_{2}(\mathrm{~mm} \mathrm{Hg})$ & $171 \pm 14$ & $172 \pm 11$ \\
$\mathrm{P}_{\mathrm{a}} \mathrm{O}_{2}(\mathrm{~mm} \mathrm{Hg})$ & $20.1 \pm 0.5$ & $19.0 \pm 0.6$ \\
{$\left[\mathrm{HCO}_{3}{ }^{-}\right](\mathrm{mmol} / \mathrm{L})$} & & \\
\hline
\end{tabular}

$* P<0.05$ for GK vs. Wistar rats

h

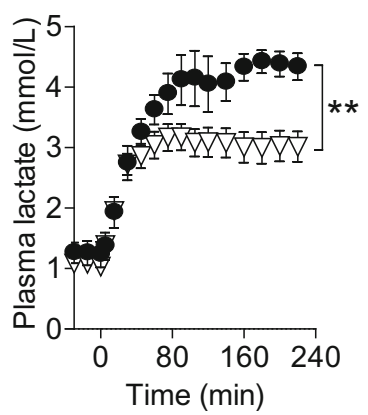

i

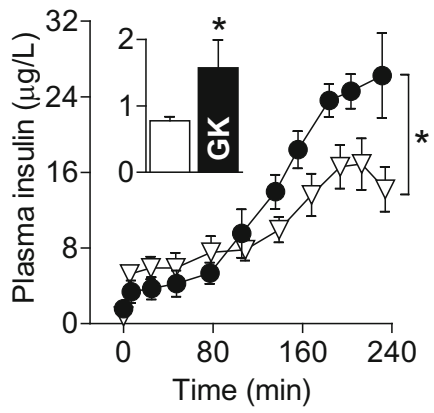

of this hyperglycemic clamp, GK rats required lower glucose infusion rate (g), showed higher plasma lactate concentration (h), and displayed higher insulin levels (i) relative to Wistar rats. The inset in panel $\mathrm{i}$ represents baseline insulin $(t=0)$. Data are mean \pm SEM. Open bars/symbols represent Wistar rats. Filled bars/symbols represent $\mathrm{GK}$ rats. $* P<0.05$, $* * P<0.01 ; * * * P<0.001$ for GK vs. Wistar rats

$0.38 \pm 0.02 \mu$ mol.g. $\min ^{-1}(P=0.041)$ in Wistar and GK rats, respectively. The resulting $T_{\max } / \mathrm{CMR}_{\mathrm{glc}}$ was $2.6 \pm 0.2$ for controls and $2.4 \pm 0.1$ for GK rats, which are similar to the ratios calculated at steady-state. Since the estimation of $K_{\mathrm{t}}$ was associated with a large uncertainty, we tested the impact of this parameter in estimating $T_{\max }$ and $\mathrm{CMR}_{\mathrm{glc}}$ (Fig. 3c): while $T_{\max }$ increased with $K_{\mathrm{t}}$, values of $K_{\mathrm{t}}$ up to $9 \mathrm{mmol} / \mathrm{L}$ were devoid of substantial effect on $\mathrm{CMR}_{\text {glc }}$.

The concentrations of amino acids required for mathematical modeling were determined by ${ }^{1} \mathrm{H}$ MRS prior to the $\left[1,6-{ }^{13} \mathrm{C}\right]$ glucose infusion. Concentrations in the brain of Wistar and GK rats were (in $\mu \mathrm{mol} / \mathrm{g}$ ), respectively, $7.6 \pm 0.3$ and $7.5 \pm 0.4$ for glutamate, $3.8 \pm 0.3$ and $3.4 \pm 0.3$ for glutamine, and $2.1 \pm 0.2$ and $2.2 \pm 0.2$ for aspartate. The mathematical model described well the incorporation of ${ }^{13} \mathrm{C}$ into these amino acids in both Wistar $\left(R^{2}=0.990, f=3390\right)$ and GK ( $R=0.994, f=5610)$ rats (Fig. 4$)$, and fluxes of oxidative metabolism in neurons and astrocytes were estimated (Table 2). Relative to controls, GK rats displayed reduced glutamate-glutamine cycle rate, depicted by lower $V_{\mathrm{NT}}$ $(-40 \%, P<0.001)$ and $V_{\mathrm{GS}}(-32 \%, P<0.001)$, and neuronal oxidative metabolism, represented by $V_{\mathrm{TCA}}{ }^{\mathrm{n}}(-7 \%$, $P=0.036)$. In turn, in the glial compartment, $V_{\mathrm{g}}$ was $33 \%$ larger in GK than Wistar rats $(P=0.011)$, but $V_{\mathrm{PC}}$ was similar 
Fig. $2{ }^{13} \mathrm{C}$ MRS at $14.1 \mathrm{~T}$ in the brain of a GK rat during $\left[1,6-{ }^{13} \mathrm{C}\right]$ glucose infusion. Spectra were acquired with a temporal resolution of $5.3 \mathrm{~min}$ for about $5 \mathrm{~h}$ (a). Spectra are displayed with a LorentzianGaussian apodization $(\mathrm{lb}=7$, $\mathrm{gf}=0.08$, gfs $=0.02$ ). The MRI slice shows the VOI location in the rat brain. b The last eight spectra from two typical experiments were summed to depict the different labeling in Wistar (left) and GK (right) rats. Spectra in panel $\mathbf{b}$ were processed with Gaussian apodization ( $\mathrm{gf}=0.08$ and $\mathrm{gfs}=0.02$ ). Labeling of carbon resonances: Glu glutamate, Gln glutamine, Asp aspartate

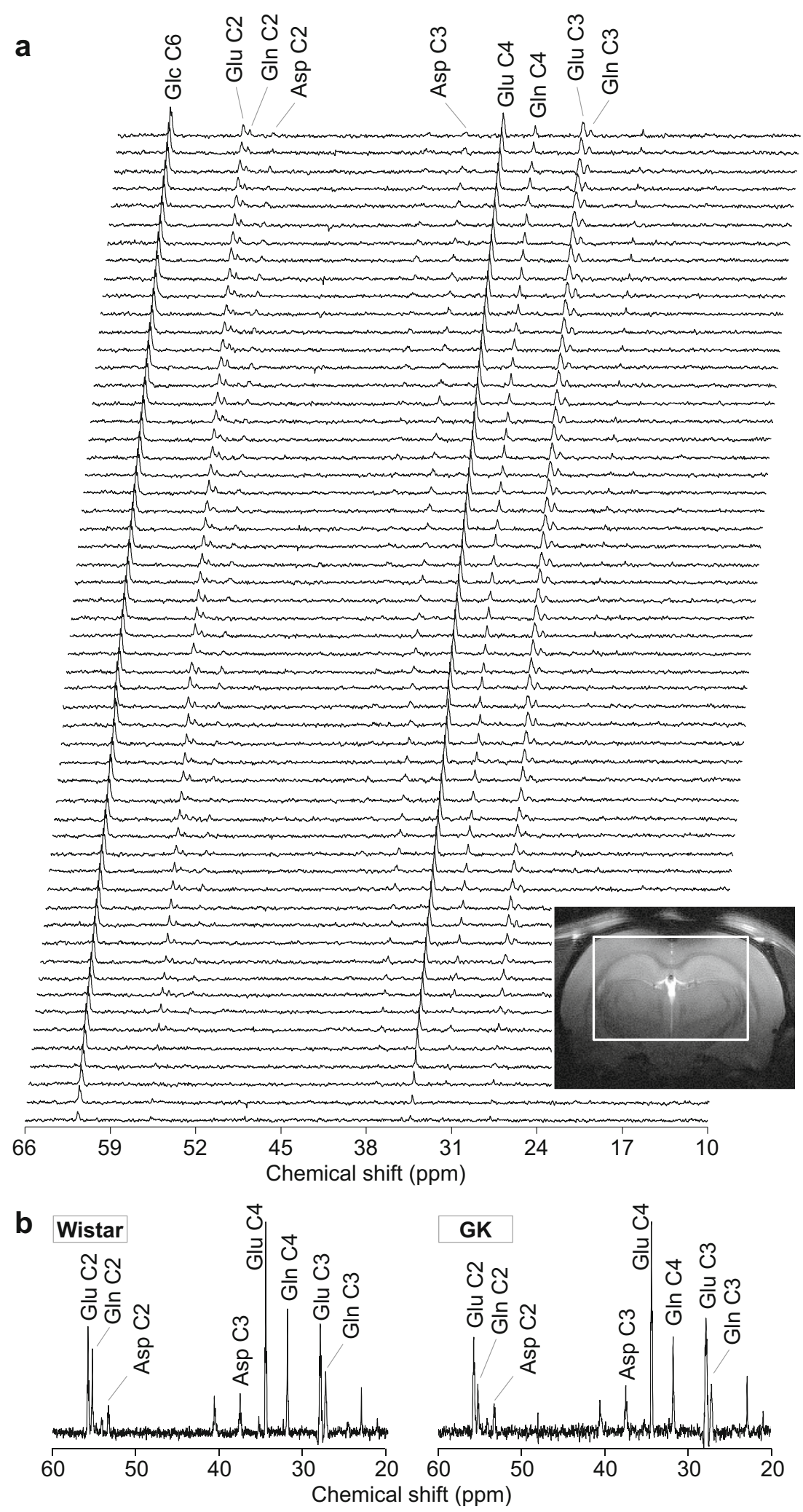

in both groups, leading to a $21 \%$ increase in $V_{\mathrm{TCA}}{ }^{\mathrm{g}}$ $(P=0.042)$. The global rate of oxidative metabolism, that is $\mathrm{CMR}_{\mathrm{glc}(\mathrm{ox})}$ (in glucose equivalents), was similar in $\mathrm{GK}$ and Wistar rats.

\section{Discussion}

The several proposed mechanisms by which T2D leads to cognitive decline include detrimental effects of oxidative 

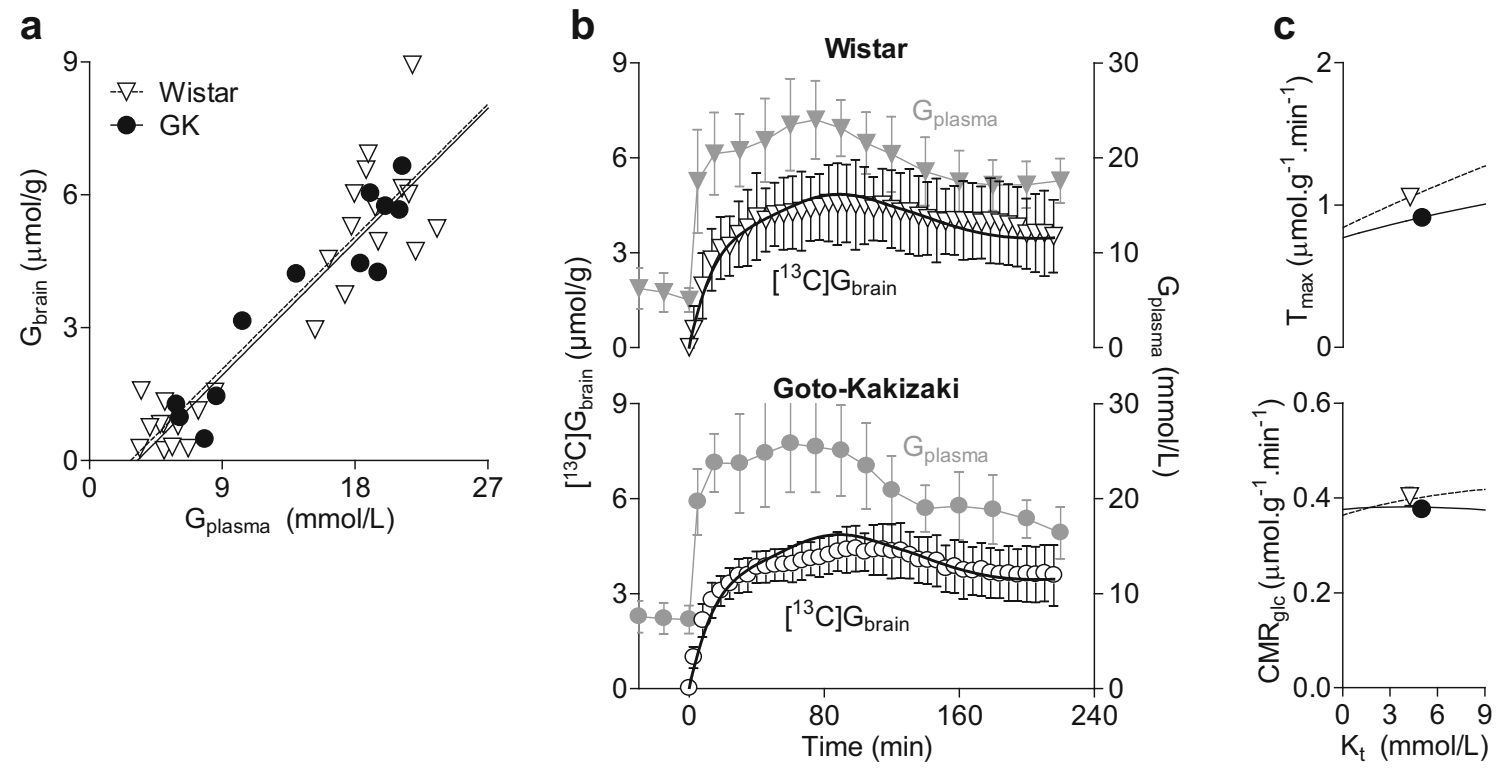

Fig. 3 Brain glucose concentration at steady-state was similar in Wistar (open triangles) and GK (filed circles) rats (a). Brain glucose concentrations at glycemia below $15 \mathrm{mmol} / \mathrm{L}$ were measured by ${ }^{1} \mathrm{H}$ MRS in vivo before $\left[1,6-{ }^{13} \mathrm{C}\right]$ glucose infusion. At plasma glucose above $15 \mathrm{mmol} / \mathrm{L}$, brain glucose concentrations were calculated from $\left[{ }^{13} \mathrm{C}\right]$ glucose measured in vivo by ${ }^{13} \mathrm{C}$ MRS and plasma $\mathrm{FE}$, assuming that brain and plasma FE are similar. The reversible Michaelis-Menten model fit is represented by dashed and solid lines for Wistar and GK rats,

respectively, and allowed $K_{\mathrm{t}}$ determination. Panel b shows the dynamic analysis of brain glucose transport and consumption using plasma glucose $\left(G_{\text {plasma }}\right.$, filled gray symbols) and its $\mathrm{FE}$, and brain $\left[{ }^{13} \mathrm{C}\right]$ glucose $\left(\left[{ }^{13} \mathrm{C}\right] G_{\text {brain }}\right.$, open symbols). The best fit of the model is represented by the solid line over $\left[{ }^{13} \mathrm{C}\right] G_{\text {brain. }}$. Panel $\mathbf{c}$ shows the effect of $K_{\mathrm{t}}$ on the determination of $T_{\mathrm{max}}$ and $\mathrm{CMR}_{\mathrm{glc}}$ by the dynamic analysis of glucose labeling. Data are mean $\pm \mathrm{SD}$
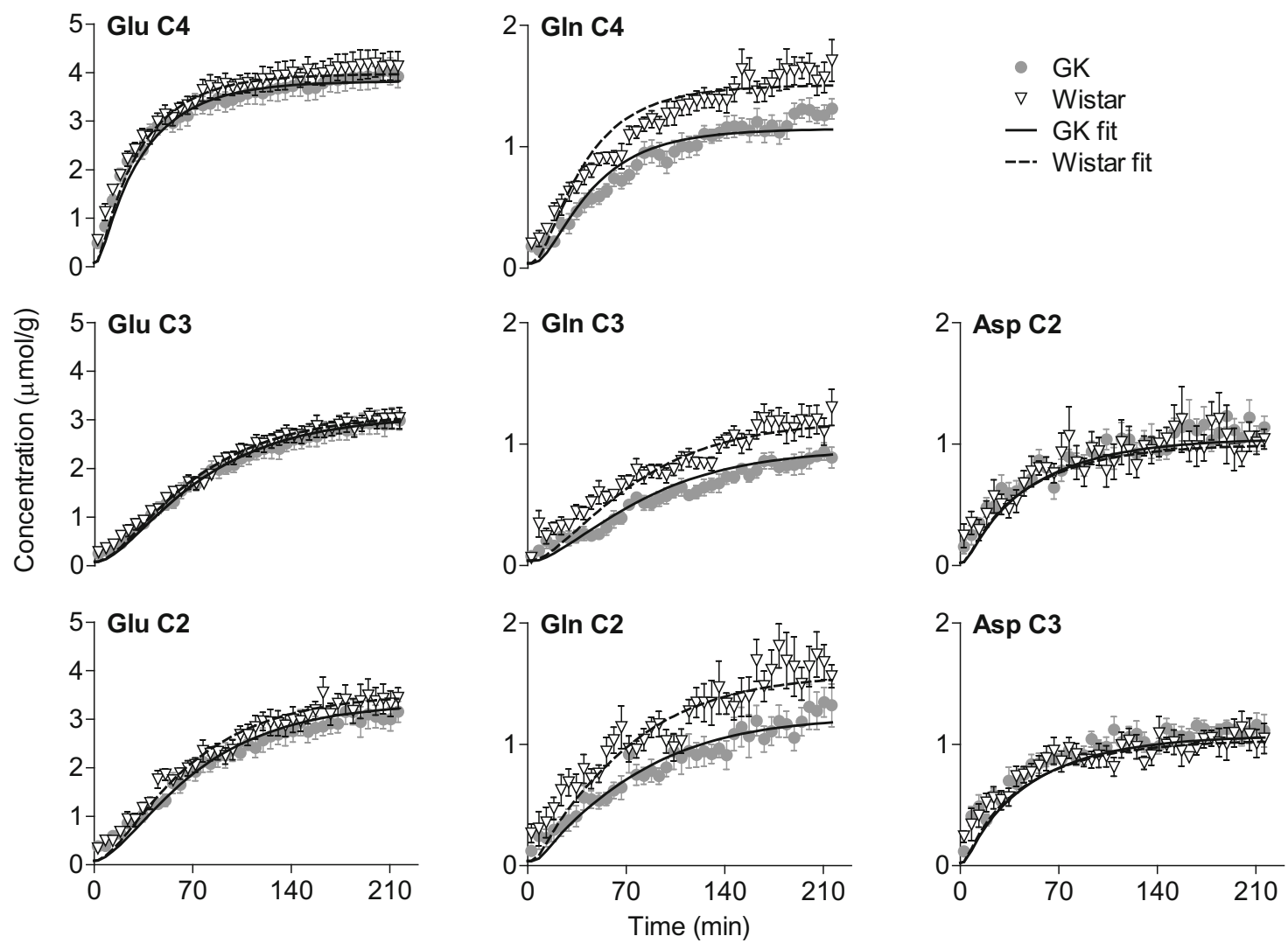

Fig. 4 Concentration of ${ }^{13} \mathrm{C}$ in aliphatic carbons of glutamate (Glu), glutamine (Gln), and aspartate (Asp) detected in the brain of GK (dots)

two-compartment model of brain energy metabolism for GK and Wistar and Wistar (triangles) rats. Solid and dashed lines represent best fit of the rats, respectively. Data are mean \pm SEM 
Table 2 Metabolic fluxes (in $\mu \mathrm{mol} / \mathrm{g} / \mathrm{min}$ ) in GK and Wistar rats

\begin{tabular}{lll}
\hline & Wistar & GK \\
\hline Measured: & & \\
$V_{\mathrm{NT}}$ & $0.20 \pm 0.02$ & $0.12 \pm 0.02 * * *$ \\
$V_{\mathrm{TCA}}{ }^{\mathrm{n}}$ & $0.34 \pm 0.01$ & $0.31 \pm 0.02 *$ \\
$V_{\mathrm{g}}$ & $0.24 \pm 0.05$ & $0.32 \pm 0.05 *$ \\
$V_{\mathrm{PC}}$ & $0.09 \pm 0.01$ & $0.08 \pm 0.01$ \\
$V_{\mathrm{X}}{ }^{\mathrm{n}}$ & $0.60 \pm 0.06$ & $0.51 \pm 0.09$ \\
$V_{\mathrm{X}}{ }^{\mathrm{g}}$ & $0.08 \pm 0.04$ & $0.07 \pm 0.04$ \\
$V_{\mathrm{dil}}$ & $0.93 \pm 0.27$ & $1.42 \pm 1.10^{\mathrm{a}}$ \\
$V_{\text {out }}$ & $0.10 \pm 0.06$ & $0.06 \pm 0.06$ \\
$T_{\text {max }}$ & $1.06 \pm 0.04$ & $0.91 \pm 0.03 * * *$ \\
$\mathrm{CMR}_{\text {glc }}$ & $0.40 \pm 0.02$ & $0.38 \pm 0.02 *$ \\
Calculated: & & \\
$V_{\mathrm{TCA}}{ }^{\mathrm{g}}$ & $0.32 \pm 0.05$ & $0.38 \pm 0.05 *$ \\
$V_{\mathrm{GS}}$ & $0.29 \pm 0.02$ & $0.20 \pm 0.03 * * *$ \\
$V_{\text {in }}$ & $0.05 \pm 0.08$ & $0.09 \pm 0.08$ \\
$\mathrm{CMR}_{\text {glc(ox })}$ & $0.37 \pm 0.03$ & $0.39 \pm 0.03$ \\
\hline
\end{tabular}

Fluxes are shown with associated SD estimated from Monte-Carlo simulations

$* P<0.05, * * * P<0.001$ after correction for multiple comparisons

${ }^{\text {a }}$ The histogram of $V_{\text {dil }}$ distribution in Monte-Carlo simulations for GK rats had a long tail, resulting in $\mathrm{SD}=7.08$. Thus, $\mathrm{SD}$ calculation excluded values that were fivefold above the estimated flux value (94\% were retained)

stress on cellular components, synaptic dysfunction, degeneration of neuronal processes or even apoptosis, neuroinflammation, and astrogliosis (Duarte 2015). These events are likely associated to metabolic alterations, which we investigated by ${ }^{13} \mathrm{C}$ MRS in vivo at high field using an optimized protocol that provides higher precision in metabolic flux determination when compared to past studies (Duarte et al. 2011), and allows to detect small flux variations in the rat brain (Sonnay et al. 2016). The present study demonstrates that insulin resistance is associated to important alterations of brain energy metabolism that are measurable non-invasively by MRS. Namely, we observed reduced neuronal TCA cycle $\left(V_{\mathrm{TCA}}{ }^{\mathrm{n}}\right)$, impaired glutamate-glutamine cycle $\left(V_{\mathrm{NT}}\right)$ and glutamine synthesis $\left(V_{\mathrm{GS}}\right)$, and exacerbated astroglial TCA cycle $\left(V_{\mathrm{TCA}}^{\mathrm{g}}\right)$ in GK rats, when compared to controls.

At steady-state $T_{\max } / \mathrm{CMR}_{\mathrm{glc}}$ in GK rats remained similar to those in the brain of controls, which apparently indicates adequate brain glucose homeostasis. Indeed, brain glucose concentration in function of glycemia in GK rats was similar to controls, as it had been previously observed in the hippocampus and cortex of streptozotocin-induced diabetic rats, a model of type 1 diabetes with chronic hyperglycemia and hypo-insulinemia (Duarte et al. 2009; Wang et al. 2012). However, analysis of brain glucose ${ }^{13} \mathrm{C}$ labeling curves revealed significantly lower $T_{\max }$ and $\mathrm{CMR}_{\mathrm{glc}}$ in
GK than Wistar rats, which suggests a global glucose hypo-metabolism, as occurs in neurodegenerative disorders (Duarte 2015). Indeed, with the parameters determined in this study, we estimate that the net brain glucose uptake at a typical plasma glucose of $7 \mathrm{mmol} / \mathrm{L}$ is $0.36 \pm 0.01$ and $0.44 \pm 0.02 \mu \mathrm{mol} / \mathrm{g} / \mathrm{min}$ for GK and Wistar rats, respectively. A reduced glucose uptake by the brain might be compensated by the utilization of other substrates (Duarte et al. 2011), such as lactate that was elevated in the blood of GK rats (versus controls) upon glucose administration, when compared to controls. In fact, total oxidative metabolism, $\mathrm{CMR}_{\text {glc(ox) }}$, was similar in both groups. Nevertheless, since GK rats display sustained hyperglycemia, their brains are exposed to excessive glucose levels when compared to normoglycemic rats, which may trigger a plethora of deleterious effects leading to neurodegeneration, namely through oxidative stress and non-enzymatic glycation of macromolecules (Baydas et al. 2003; Ates et al. 2007).

While the global oxidation rate was unaltered relative to controls, the GK rats displayed a shift towards higher metabolic rates in glial cells, at the expense of oxidative metabolism in neuronal mitochondria. In particular, the relative contribution of the astrocytic TCA cycle for whole brain oxidative metabolism was $50 \%$ in GK rats, while it was only about $40 \%$ in Wistar rats, as well as in previous studies in the rat brain (Duarte et al. 2011; Duarte and Gruetter 2013; Sonnay et al. 2016, 2017a). The diabetes-induced reduction in neuronal oxidative metabolism paralleled a reduction in the rate of the glutamate-glutamine cycle $\left(V_{\mathrm{NT}}\right.$ and $\left.V_{\mathrm{GS}}\right)$ that depicts glutamatergic neurotransmission. In line with impaired neuronal oxidative metabolism and neurotransmission, GK rats were reported to exhibit a loss of cortical neurons (Hussain et al. 2014), altered expression of genes involved in neurotransmission in the cortex (Abdul-Rahman et al. 2012), and reduced dendritic spine density and neuronal viability in CA1 of the hippocampus ( $\mathrm{Li}$ et al. 2013), when compared to non-diabetic Wistar rats. The brain of GK rats was also reported to display reduced survival of neural progenitor cells, despite increased proliferation in both the dentate gyrus and the subventricular zone, when compared to Wistar rats (Lang et al. 2009). On the other hand, in accordance with exacerbated mitochondrial metabolism in glial cells, there have been reports suggesting the occurrence of astrogliosis and microgliosis in the brain of GK rats (Prakash et al. 2013; Hussain et al. 2014). Altogether, these reports point to neuropathological features appearing after 3 months of age in the GK rat model of T2D (Lang et al. 2009; Abdul-Rahman et al. 2012; Li et al. 2013; Prakash et al. 2013; Hussain et al. 2014). Learning and memory performance was reported in 7-month-old GK rats, the age of our study (Li et al. 2013). Both nerve terminal degeneration and astrogliosis were also reported in other animal models displaying insulin resistance. Namely, the hippocampus of obese, diabetic NONcNZO10/LtJ mice display decreased 
density of nerve terminal proteins required for synaptic transmission and plasticity, as well as increased astrocyte reactivity (Duarte et al. 2012). In addition, diabetic BBZDR/Wor rats show evidence of neuronal degeneration, reduced presynaptic densities, and astrogliosis in the frontal cortex (Li et al. 2007).

Few studies have investigated brain energy metabolism in T2D. Leffa et al. fed male Swiss mice for 13 weeks on either standard chow or a cafeteria diet, the latter developing insulin resistance (Leffa et al. 2017). Cafeteria diet consumption led to reduced activity of citrate synthase in the prefrontal cortex, hippocampus, and hypothalamus, as well as reduced activity of respiratory chain complexes in several brain areas (Leffa et al. 2017). Although this study did not identify the cellular populations displaying such metabolic alterations, one might speculate that reduced oxidative metabolism in mice exposed to the cafeteria diet is in the neurons, the most active cells in the brain. Another study reported that Zucker diabetic fatty (ZDF) and Zucker obese rats displayed reduced ${ }^{13} \mathrm{C}$ labeling incorporation from $\left[1-{ }^{13} \mathrm{C}\right]$ glucose into cortical and hippocampal glutamate (amino acid primarily formed and located in neurons), compared to lean controls (Sickmann et al. 2010). Accordingly, the brain of ZDF rats was shown to display reduced activity of respiratory chain complexes, resulting in reduced ATP synthesis (Raza et al. 2015).

Aiming at measuring glial metabolism, Zheng et al. administered $\left[2-{ }^{13} \mathrm{C}\right]$ acetate to either diabetic $d b / d b$ mice or controls, and then evaluated ${ }^{13} \mathrm{C}$ labeling incorporation into metabolites involved in oxidative metabolism in brain extracts (Zheng et al. 2017). Labeling incorporation from $\left[2-{ }^{13} \mathrm{C}\right]$ acetate into glutamate, glutamine, GABA, and the TCA cycle intermediate succinate was significantly decreased in $d b / d b$ relative to wild-type mice. Since acetate is predominantly metabolized in astrocytes (Lanz et al. 2013 and references therein), this finding appears in contradiction with the results now obtained in GK rats indicating increased astrocytic metabolism in T2D. However, the results by Zheng et al. were obtained with a single-point measurement of the ${ }^{13} \mathrm{C}$ labeling (Zheng et al. 2017), and thus could be affected by the brain catabolism of unlabeled substrates, namely glucose and ketones. These brain substrates are plentifully available in the $d b / d b$ mouse model, can be taken by the brain, and produce unlabeled acetyl-CoA that competes with $\left[2-{ }^{13} \mathrm{C}\right]$ acetate-derived molecules for citrate synthase (Andersen et al. 2017). In our study, by measuring the time course of ${ }^{13} \mathrm{C}$ isotope incorporation into metabolites, we are effectively able to determine metabolic fluxes of brain metabolism. In agreement with our results, increased relative contribution of astrocytic oxidative metabolism to the support of glutamatergic neurotransmission was found in streptozotocin-induced diabetic rats (García-Espinosa et al. 2003). Also in line with exacerbated astrocytic oxidative metabolism in diabetes, larger brain acetate utilization was found in patients with type 1 diabetes than in healthy subjects (Mason et al. 2006).
Major features of the diabetic phenotype in GK rats are a mild hyperglycemia and insulin resistance, and the present study cannot dissociate the contributions of these alterations to the observed metabolic changes in the brain. Insulin regulates a plethora of cellular events required for adequate brain functioning, including synaptic activity and plasticity (Kleinridders et al. 2014). Therefore, in addition to glucose neurotoxicity, impaired brain insulin signaling is likely an important component of the cognitive decrements in diabetes (Rönnemaa et al. 2008; Duarte 2015). Moreover, like in Alzheimer's disease, impaired brain insulin-PI3K/Akt signaling in diabetes can induce hyperphosphorylation of the tau protein and favor $A \beta$ deposition, leading to formation of neurofibrillary tangles and amyloid plaques, thus contributing to neurodegeneration (Candeias et al. 2012). Noteworthy, IGF-1 and insulin were shown to directly regulate mitochondrial metabolism in cultured neurons and astrocytes (Sonnewald et al. 1995). To directly tackle effects of brain insulin resistance on energy metabolism, it would be of interest to establish a model of brain insulin resistance in the absence of alterations of the glycemic control.

GK rats displayed increased circulating corticosterone. It was demonstrated that activation of glucocorticoid receptors play a role in diabetes-induced brain dysfunction in both insulin-dependent and insulin-resistant animal models (Stranahan et al. 2008). Therefore, it cannot be excluded that glucocorticoid-mediated deficits in neurogenesis and synaptic plasticity also contribute for impaired oxidative metabolism in neurons in the brain of GK rats.

In the present study, glucose was infused to produce comparable glycemia and glucose FE over the course of ${ }^{13} \mathrm{C}$ MRS. However, we did not control circulating levels of insulin or corticosterone, which were higher in GK rats than in controls, and are known to regulate brain function (Stranahan et al. 2008; Kleinridders et al. 2014). Therefore, the measured metabolic alterations might reflect both the diabetes-induced degenerative process and the overall hormonal imbalance in T2D.

The physiology of the rats was carefully controlled during MRS experiments, and maintained within normal values. Nevertheless, GK rats displayed lower $\mathrm{P}_{\mathrm{a}} \mathrm{CO}_{2}$ than controls. Although $\mathrm{P}_{\mathrm{a}} \mathrm{CO}_{2}$ impacts cerebral blood flow and volume, the observed variation does not affect substantially nutrient provision to the brain parenchyma (Shockley and LaManna 1988), and is certainly devoid of effects on the measured metabolic parameters.

At last, it should be noted that the analysis of brain metabolism was carried out with a metabolic model of ${ }^{13} \mathrm{C}$ labeling that relies on a number of assumptions, which are supported by biochemical evidence (Sonnay et al., 2017b). Most importantly, the relative distribution of glutamate between neurons and astrocytes can influence flux estimations, namely of 
$V_{\mathrm{TCA}}{ }^{\mathrm{n}}$ and $V_{\mathrm{TCA}}{ }^{\mathrm{g}}$. However, the concentration of brain glutamate was similar in GK and Wistar rats, and there is no evidence of diabetes-induced changes in the neuron-to-glia glutamate proportion.

In conclusion, the present study demonstrates that T2D in GK rats is associated with impaired neuronal oxidative metabolism and glutamatergic neurotransmission, and with enhanced astroglial oxidative metabolism.

Acknowledgements The authors are grateful to Dr. Ana F. Soares (LIFMET, EPFL) for fruitful discussions.

Funding This research was supported by the Swiss National Science Foundation (grant 148250), National Competence Center in Biomedical Imaging (NCCBI), and by Centre d'Imagerie BioMédicale (CIBM) of the UNIL, UNIGE, HUG, CHUV, EPFL, and the Leenaards and Jeantet Foundations.

Compliance with Ethical Standards All experiments were performed in accordance with the Swiss federal law on animal experimentation and approved by the local authority (EXPANIM-SCAV), and are reported according to the ARRIVE guidelines.

\section{References}

Abdul-Rahman O, Sasvari-Szekely M, Ver A et al (2012) Altered gene expression profiles in the hippocampus and prefrontal cortex of type 2 diabetic rats. BMC Genomics 13:81

Andersen JV, Christensen SK, Nissen JD, Waagepetersen HS (2017) Improved cerebral energetics and ketone body metabolism in $\mathrm{db} /$ db mice. J Cereb Blood Flow Metab 37(3):1137-1147

Ates O, Cayli SR, Yucel N et al (2007) Central nervous system protection by resveratrol in streptozotocin-induced diabetic rats. J Clin Neurosci 14:256-260

Baker LD, Cross D, Minoshima S, Belongia D, Watson GS, Craft S (2011) Insulin resistance is associated with Alzheimer-like reductions in regional cerebral glucose metabolism for cognitively impaired normal adults with pre-diabetes or early type 2 diabetes. Arch Neurol 68:51-57

Baumgart M, Snyder HM, Carrillo MC, Fazio S, Kim H, Johns H (2015) Summary of the evidence on modifiable risk factors for cognitive decline and dementia: a population-based perspective. Alzheimers Dement 11(6):718-726

Baydas G, Nedzvetskii VS, Tuzcu M, Yasar A, Kirichenko SV (2003) Increase of glial fibrillary acidic protein and S-100B in hippocampus and cortex of diabetic rats: effects of vitamin E. Eur J Pharmacol 462:67-71

Biessels GJ, Staekenborg S, Brunner E, Brayne C, Scheltens P (2006) Risk of dementia in diabetes mellitus: a systematic review. Lancet Neurol 5(1):64-74

Calvo-Ochoa E, Hernández-Ortega K, Ferrera P, Morimoto S, Arias C (2014) Short-term high-fat-and-fructose feeding produces insulin signaling alterations accompanied by neurite and synaptic reduction and astroglial activation in the rat hippocampus. J Cereb Blood Flow Metab 34(6):1001-1008

Candeias E, Duarte AI, Carvalho C et al (2012) The impairment of insulin signaling in Alzheimer's disease. IUBMB Life 64(12):951-957

Candeias E, Duarte AI, Sebastião I, Fernandes MA, Plácido AI, Carvalho C, Correia S, Santos RX, Seiça R, Santos MS, Oliveira CR, Moreira PI (2016) Middle-aged diabetic females and males present distinct susceptibility to Alzheimer disease-like pathology. Mol Neurobiol. https://doi.org/10.1007/s12035-016-0155-1

Duarte JMN (2015) Metabolic alterations associated to brain dysfunction in diabetes. Aging Dis 6(5):304-321

Duarte JMN, Agostinho PM, Carvalho RA, Cunha RA (2012) Caffeine consumption prevents diabetes-induced memory impairment and synaptotoxicity in the hippocampus of NONcNZO10/LtJ mice. PLoS One 7:e21899

Duarte JMN, Carvalho RA, Cunha RA, Gruetter R (2009) Caffeine consumption attenuates neurochemical modifications in the hippocampus of streptozotocin-induced diabetic rats. J Neurochem 111(2): 368-379

Duarte JMN, Cunha RA, Carvalho RA (2007) Different metabolism of glutamatergic and GABAergic compartments in superfused hippocampal slices characterized by nuclear magnetic resonance spectroscopy. Neurosci 144:1305-1313

Duarte JMN, Gruetter R (2012) Characterization of cerebral glucose dynamics in vivo with a four-state conformational model of transport at the blood-brain-barrier. J Neurochem 121:396-406

Duarte JMN, Gruetter R (2013) Glutamatergic and GABAergic energy metabolism measured in the rat brain by ${ }^{13} \mathrm{C}$ NMR spectroscopy at 14.1 T. J Neurochem 126(5):579-590

Duarte JMN, Lanz B, Gruetter R (2011) Compartmentalised cerebral metabolism of $\left[1,6-{ }^{13} \mathrm{C}\right]$ glucose determined by in vivo ${ }^{13} \mathrm{C} \mathrm{NMR}$ spectroscopy at $14.1 \mathrm{~T}$. Front Neuroenergetics $3: 3$

Francis GJ, Martinez JA, Liu WQ et al (2008) Intranasal insulin prevents cognitive decline, cerebral atrophy and white matter changes in murine type I diabetic encephalopathy. Brain 131:3311-3334

García-Espinosa MA, García-Martín ML, Cerdán S (2003) Role of glial metabolism in diabetic encephalopathy as detected by high resolution ${ }^{13} \mathrm{C}$ NMR. NMR Biomed 16(6-7):440-449

Geijselaers SL, Sep SJ, Stehouwer CD, Biessels GJ (2015) Glucose regulation, cognition, and brain MRI in type 2 diabetes: a systematic review. Lancet Diabetes Endocrinol 3(1):75-89

Henry PG, Tkác I, Gruetter R (2003) ${ }^{1} \mathrm{H}$-localized broadband ${ }^{13} \mathrm{C}$ NMR spectroscopy of the rat brain in vivo at $9.4 \mathrm{~T}$. Magn Reson Med 50: 684-692

Hussain S, Mansouri S, Sjöholm Å, Patrone C, Darsalia V (2014) Evidence for cortical neuronal loss in male type 2 diabetic GotoKakizaki rats. J Alzheimers Dis 41(2):551-660

Kapogiannis D, Mattson MP (2011) Disrupted energy metabolism and neuronal circuit dysfunction in cognitive impairment and Alzheimer's disease. Lancet Neurol 10:187-198

Kleinridders A, Ferris HA, Cai W, Kahn CR (2014) Insulin action in brain regulates systemic metabolism and brain function. Diabetes 63(7): 2232-2243

Lang BT, Yan Y, Dempsey RJ, Vemuganti R (2009) Impaired neurogenesis in adult type-2 diabetic rats. Brain Res 1258:25-33

Lanz B, Gruetter R, Duarte JMN (2013) Metabolic flux and compartmentation analysis in the brain in vivo. Front Endocrinol (Lausanne) 4: 156

Leffa DD, Rezin GT, Daumann F et al (2017) Effects of Acerola (Malpighia emarginata DC.) juice intake on brain energy metabolism of mice fed a cafeteria diet. Mol Neurobiol 54(2):954-963

Li XH, Xin X, Wang Y et al (2013) Pentamethylquercetin protects against diabetes-related cognitive deficits in diabetic Goto-Kakizaki rats. J Alzheimers Dis 34(3):755-767

Li ZG, Zhang W, Sima AA (2007) Alzheimer-like changes in rat models of spontaneous diabetes. Diabetes 56(7):1817-1824

Mason GF, Petersen KF, Lebon V, Rothman DL, Shulman GI (2006) Increased brain monocarboxylic acid transport and utilization in type 1 diabetes. Diabetes 55(4):929-934

Messier C (2005) Impact of impaired glucose tolerance and type 2 diabetes on cognitive aging. Neurobiol Aging 26(Suppl 1):26-30

Moheet A, Mangia S, Seaquist ER (2015) Impact of diabetes on cognitive function and brain structure. Ann N Y Acad Sci 1353:60-71 
Moreira PI, Santos MS, Seiça R, Oliveira CR (2007) Brain mitochondrial dysfunction as a link between Alzheimer's disease and diabetes. J Neurol Sci 257(1-2):206-214

Nitta A, Murai R, Suzuki N et al (2002) Diabetic neuropathies in brain are induced by deficiency of BDNF. Neurotoxicol Teratol 24:695-701

Palta P, Schneider AL, Biessels GJ, Touradji P, Hill-Briggs F (2014) Magnitude of cognitive dysfunction in adults with type 2 diabetes: a meta-analysis of six cognitive domains and the most frequently reported neuropsychological tests within domains. J Int Neuropsychol Soc 20(3):278-291

Portha B (2005) Programmed disorders of beta-cell development and function as one cause for type 2 diabetes? The GK rat paradigm. Diabetes Metab Res Rev 21(6):495-504

Prakash R, Johnson M, Fagan SC, Ergul A (2013) Cerebral neovascularization and remodeling patterns in two different models of type 2 diabetes. PLoS One 8(2):e56264

Raza H, John A, Howarth FC (2015) Increased oxidative stress and mitochondrial dysfunction in zucker diabetic rat liver and brain. Cell Physiol Biochem 35(3):1241-1251

Rönnemaa E, Zethelius B, Sundelöf J et al (2008) Impaired insulin secretion increases the risk of Alzheimer disease. Neurology 71:10651071

Saravia FE, Revsin Y, Gonzalez Deniselle MC et al (2002) Increased astrocyte reactivity in the hippocampus of murine models of type 1 diabetes: the nonobese diabetic (NOD) and streptozotocin-treated mice. Brain Res 957:345-353

Shockley RP, LaManna JC (1988) Determination of rat cerebral cortical blood volume changes by capillary mean transit time analysis during hypoxia, hypercapnia and hyperventilation. Brain Res 454(1-2): $170-178$

Sickmann HM, Waagepetersen HS, Schousboe A, Benie AJ, Bouman SD (2010) Obesity and type 2 diabetes in rats are associated with altered brain glycogen and amino-acid homeostasis. J Cereb Blood Flow Metab 30(8):1527-1537

Sonnay S, Duarte JMN, Just N, Gruetter R (2016) Compartmentalised energy metabolism supporting glutamatergic neurotransmission in response to increased activity in the rat cerebral cortex: a ${ }^{13} \mathrm{C}$ MRS study in vivo at 14.1 T. J Cereb Blood Flow Metab 36(5):928-940

Sonnay S, Duarte JMN, Just N, Gruetter R (2017a) Energy metabolism in the rat cortex under thiopental anaesthesia measured in vivo by ${ }^{13} \mathrm{C}$ MRS. J Neurosci Res in press doi. https://doi.org/10.1002/jnr.24032

Sonnay S, Gruetter R, Duarte JMN (2017b) How energy metabolism supports cerebral function: insights from ${ }^{13} \mathrm{C}$ magnetic resonance studies in vivo. Front Neurosci 11:288

Sonnewald U, Wang AY, Petersen SB et al (1995) ${ }^{13}$ C NMR study of IGF-I- and insulin-effects on mitochondrial function in cultured brain cells. Neuroreport 6(6):878-880

Spauwen PJ, Köhler S, Verhey FR, Stehouwer CD, van Boxtel MP (2013) Effects of type 2 diabetes on 12-year cognitive change: results from the Maastricht Aging Study. Diabetes Care 36(6):15541561

Stranahan AM, Arumugam TV, Cutler RG, Lee K, Egan JM, Mattson MP (2008) Diabetes impairs hippocampal function through glucocorticoid-mediated effects on new and mature neurons. Nat Neurosci 11(3):309-317

Tomlinson DR, Gardiner NJ (2008) Glucose neurotoxicity. Nat Rev Neurosci 9:36-45

van den Berg E, Dekker JM, Nijpels G et al (2008) Cognitive functioning in elderly persons with type 2 diabetes and metabolic syndrome: the Hoorn study. Dement Geriatr Cogn Disord 26(3):261-269

van der Graaf M, Janssen SW, van Asten JJ et al (2004) Metabolic profile of the hippocampus of Zucker Diabetic Fatty rats assessed by in vivo ${ }^{1} \mathrm{H}$ magnetic resonance spectroscopy. NMR Biomed 17(6):405-410

Wang WT, Lee P, Yeh HW, Smirnova IV, Choi IY (2012) Effects of acute and chronic hyperglycemia on the neurochemical profiles in the rat brain with streptozotocin-induced diabetes detected using in vivo ${ }^{1} \mathrm{H}$ MR spectroscopy at 9.4 T. J Neurochem 121(3):407-417

Zheng H, Zheng Y, Wang D et al (2017) Analysis of neuron-astrocyte metabolic cooperation in the brain of $\mathrm{db} / \mathrm{db}$ mice with cognitive decline using ${ }^{13} \mathrm{C}$ NMR spectroscopy. J Cereb Blood Flow Metab 37(1):332-343 